\title{
DISCURSOS Y ESTRATEGIAS EN LOS GRUPOS SIKURIS FEMENINOS EN EL SUR PERUANO: LA EXPERIENCIA DE WARMI SIKURI AWKA SISA EN AREQUIPA, PERÚ (2018-2019)
}

\author{
DISCOURSES AND STRATEGIES IN FEMALE SIKURI GROUPS IN SOUTHERN \\ PERU: THE EXPERIENCE OF WARMI SIKURI AWKA SISA IN AREQUIPA, PERU
} (2018-2019)

\author{
Verónica García Jarufe ${ }^{*}$ y Zoila Elena Vega Salvatierra**
}

\begin{abstract}
Algunas sociedades andinas han asignado roles de género a prácticas musicales específicas, como es el caso de los grupos de sikuris en el altiplano andino, que están integrados exclusivamente por intérpretes masculinos en sus lugares de origen. La migración y los desplazamientos poblacionales intensos de finales del siglo XX y comienzos del XXI desde esta zona hacia otras ciudades como Arequipa, en el sur peruano, han motivado cambios en la conformación de estos grupos en donde se observa la creciente presencia de integrantes femeninas y la conformación de conjuntos exclusivamente femeninos, subvirtiendo así el orden establecido por las comunidades en los lugares de origen. Las mujeres buscan y emplean discursos fundamentados en nuevas ideologías y construcciones de pensamiento que les permiten justificar esta subversión de la tradición establecida y diversifican notoriamente la acción de los grupos sikuris incursionando en actividades muy distintas de las que originalmente se asociaba a la participación de estos grupos. El presente estudio aborda el impacto que ha tenido la incorporación de mujeres en los grupos de sikuris en la ciudad de Arequipa, sus estrategias de asimilación, la participación de dichos grupos mixtos o femeninos en actividades de interacción social de tipo religioso o recreativo, así como las restricciones, desigualdades y otros avatares en el ejercicio de su actividad, empleando para ello los conceptos de dinamismo cultural, estudios de género, tradición y cambio en los comportamientos sociales asociados a la música, mediante el estudio del grupo Warmi Sikuri Awka Sisa, primer grupo enteramente femenino conformado en dicha ciudad.
\end{abstract}

Palabras claves: Mujer sikuri, migración, música andina, discursos feministas y música.

Some Andean societies have assigned gender roles to specific musical practices, as is the case of sikuri groups in the Andean highlands, which are made up exclusively of male performers in their places of origin. Migration and intense population displacements of the late Twentieth and early Twenty-first centuries from this area to other cities such as Arequipa, in southern Peru, have led to changes in the makeup of these groups in which the growing presence of female members is observed. The formation of exclusively female groups, thus subverting the order established by the communities in the places of origin. Women seek and use discourses based on new ideologies and constructions of thought that allow them to justify this subversion of the established tradition and notably diversify Sikuris groups' actions, which are engaged in very different activities from those originally associated with the participation of these groups. This study addresses the impact that the incorporation of women into sikuri groups in the city of Arequipa has had, their assimilation strategies, the participation of mixed or female groups in religious or recreational and social interaction activities, as well as restrictions, inequalities, and other vicissitudes in the exercise of their activity. Using the concepts of cultural dynamism, gender studies, tradition, and change in social behaviors associated with music, through the study of the Warmi Sikuri Awka Sisa group, the first entirely female group formed in that city.

Key words: Female sikuri, migration, Andean music, music and feminist discourses.

\section{Introducción}

En la región de Puno, Perú, las actividades performativas de los conjuntos Sikuris se guían por determinadas reglamentaciones (o convenciones) colectivas respecto de la participación de roles de géneros en tales actividades. Algunos investigadores han observado estas convenciones y han indagado sus causas y razones. Thomas Turino, en sus trabajos realizados en la comunidad de Conima, al sur de la región de Puno entre 1985 y 1986, observó que las mujeres no tocaban los sikus y sus informantes explicaron que simplemente esta actividad no era de su interés o que su participación no era parte de las costumbres locales (Turino 1992).

Esta ausencia de la mujer en la interpretación del siku en Puno podría explicarse mediante algunos mitos como el descrito por Carlos Sánchez cuando

\footnotetext{
* Universidad Nacional de San Agustín, programa de Antropología. Arequipa, Perú. Correo electrónico: vgarcia@unsa.edu.pe

** Universidad Nacional de San Agustín, Departamento Académico de Artes. Arequipa, Perú. Correo electrónico: zvega@ unsa. edu.pe
} 
entrevistó a un representante de una asociación cultural local (la Asociación Educativa y Cultural Rurarcaya), quien manifestó lo siguiente:

La mujer al soplar utiliza la boca y a través de ella insufla el aire, con el cual se va parte de su vida, por lo que su pecho se vacía de aire y se "seca", en otras palabras, si la mujer se dedica a la práctica musical pierde su condición de madre, así como su dedicación permanente al hogar, separándose de él, por ello su pecho se "seca" al desligarse de su tarea de alimentar (Sánchez 2015:306).

La asignación de roles de género en diferentes actividades en las comunidades altiplánicas en la región de Puno es una constante que puede observarse no únicamente en el desenvolvimiento de las tropas sikuris. Tal asignación de roles, según María Rostorowski, tuvo raíces muy antiguas en el mundo andino. En este espacio "El varón abría la tierra con la chakitaklla y la mujer depositaba el grano, nunca al revés" (Rostorowski 1998). Como lo explica Ana Carrasco y Vivian Gavilán, las identidades de género son procesos de interiorización de las tradiciones culturales que ayudan a configurar las prácticas sociales entre hombres y mujeres (Carrasco y Gavilán 2017), por lo que, si extrapolamos estas asignaciones de roles específicos dentro de actividades determinadas como las actuaciones de tropas sikuris en el altiplano puneño, encontraremos que hombres y mujeres tienen prescrita su participación en atención a sus características biológicas: el hombre toca y la mujer baila, ya que la fuerza musical para tocar reside en el primero, mientras que la delicadeza del baile es connatural a la segunda.

Estos roles de género explican la ausencia de mujeres en las tropas sikuris de las provincias puneñas, pero ¿qué sucede cuando el fenómeno migratorio y la difusión de contenidos locales llega a nuevos contextos y se generan transformaciones en los usos y desenvolvimientos de estos grupos? ¿Qué discursos, imaginarios e ideologías son empleados para explicar -y en ocasiones justificar- estas transformaciones?

El movimiento migratorio que se registra desde las regiones altiplánicas de la región de Puno hacia núcleos urbanos del sur de Perú como las ciudades de Tacna, Moquegua y Arequipa impulsa también la expansión del fenómeno sikuri hacia las ciudades mencionadas donde adoptan nuevos lenguajes, usos y discursos, en concordancia con los nuevos entornos, donde las poblaciones migrantes y sus descendientes entran en contacto con formas de pensamiento que les plantean nuevos desafíos.

En nuevos entornos, los contenidos culturales se transforman, pero en el caso concreto de las poblaciones altiplánicas que se desplazan a los núcleos urbanos, pueden ocurrir fenómenos de reinterpretación de estos contenidos y resignificación de los mismos. Ponciano del Pino ha observado cómo las poblaciones migrantes relacionan el entorno natural (paisaje, apus) con el poder y tradición que se gestan en esta zona. Al producirse el alejamiento del centro de producción de cultura, se trastoca esta jerarquía (Del Pino 2017). Entonces, como lo ha señalado también Teresa Rodríguez, los jóvenes migrantes tienen mayor capacidad de agencia para distanciarse de la matriz de posiciones sociales y, a veces, tienden a cuestionar el excesivo ritualismo y las obligaciones asociadas a ello (Rodríguez 2016).

En algunos casos, el fenómeno sikuri interesa a personas que han perdido todo contacto con la tradición original y que le asignan significados, propósitos y prácticas completamente diferentes, por lo que se generan nuevos sistemas de sentidos y ejercicio de la actividad. Dicha asimilación de ideologías y patrones de comportamiento distintos a los pertenecientes a los usos originales supone una respuesta a estímulos contextuales que hacen que las prácticas culturales reasignen significados y busquen dar satisfacción a necesidades surgidas en los nuevos escenarios.

Diversos investigadores han abordado la presencia, calendario y acción de los sikuris en contextos distintos a los originales procedentes del altiplano peruano, chileno y boliviano. Pablo Mardones y Fer Barragán, en su investigación de los sikuris en Buenos Aires, explican cómo los bolivianos se han apropiado de espacios públicos por medio de tres estrategias. Primero, la reproducción del aimara-quechua e indígena; segundo la creación de encuentros y festivales y tercero con su presencia en manifestaciones y marchas en apoyo y repudio a diferentes causas sociales, políticas, culturales y otras (Barragán y Mardones 2015). Esta forma de inserción en nuevos contextos se relaciona con lo explicado por Adil Podhjacser quien señala que, con la inserción de migrantes, se da una transformación entre las relaciones interculturales y revaloración de identidad, no solo boliviana sino latinoamericana 
(Podhajcer 2015). Estas adaptaciones también fueron estudiadas por Miguel Ángel Ibarra, acerca de los sikuris en Santiago de Chile, quien comenta se construyen nuevas sonoridades que representan "lo andino" y la construcción de nuevas identidades musicales con un discurso centrado en la reivindicación de lo indígena u originario (Ibarra 2016).

Estos trabajos -por mencionar solo algunosabordan nociones como la identidad y la revaloración y varios de ellos centran su atención en una constante: estos grupos regulan sus actividades públicas de acuerdo con las festividades del calendario andino.

En Perú, no obstante, la literatura se ha mostrado más estrecha en su estudio del fenómeno sikuri. Muchos estudios, llevados a cabo por etnomusicólogos norteamericanos (Cf. los trabajos de T. Turino) se han concentrado en los estudios de las tropas sikuris en sus lugares de origen, en su mayoría, en las provincias de la región de Puno (Turino 1992).

Otros estudiosos como Américo Valencia se han concentrado en describir la historia de los sikuris, su relación con la cosmovisión andina, los diferentes estilos, los espacios que ocupan los sikuris y cómo se desarrollan en fiestas tradicionales y solo en Sánchez Huaringa y la entrevista a Cotrina, mencionada líneas arriba, se nombran algunos puntos referidos al enfoque de género para el estudio de estos fenómenos en sus nuevos o viejos contextos.

Entonces surge una pregunta ¿cómo se ha dado la adaptación, reinvención y recirculación de la práctica sikuri desde la perspectiva de género en núcleos urbanos alejados o distintos de los ubicados en la zona altiplánica en el territorio peruano? ¿Cómo han podido las mujeres sikuris peruanas acceder a prácticas que les estaban vedadas o les eran ajenas en los lugares de origen de tales prácticas y qué discursos e imaginarios han sido necesarios para sustentar $-\mathrm{y}$ a veces justificar- estas novedades?

Al ser la ciudad de Arequipa un núcleo urbano importante en la Macro Región Sur del Perú, convergen en ella diversas prácticas, realidades y tradiciones que encuentran espacios y ocasiones que se transforman constantemente para satisfacer las necesidades de quienes cultivan estas expresiones. Es así que, en la última década, las agrupaciones de sikuris han experimentado una serie de cambios que incluyen la mayor participación femenina en ellos e incluso la conformación de grupos exclusivamente femeninos. No existen estudios de las transformaciones del fenómeno y práctica sikuri desde la perspectiva de género en una ciudad con un sentido identitario tan particular como lo es Arequipa, cuya población ha aumentado considerablemente en las últimas décadas a causa de la migración proveniente en su gran mayoría de la región altiplánica (así como por su propio crecimiento "natural"), por lo que consideramos que una primera aproximación a un estudio de estas características podría ofrecer indicios de cómo se transforman estas prácticas en entornos diferentes a los originales, especialmente los referidos a los roles de género.

Juan Chacama y Alberto Díaz explican que los contextos arqueológicos evidencian la utilidad y significación de los instrumentos musicales como artefactos culturales tanto en prácticas festivas como en actividades culturares circunscritas a ritos mortuorios (Chacama y Díaz 2011). Asimismo Guillermo Focacci y Elías Pizarro comentan que existió estrecha relación entre el uso de instrumentos y manifestaciones ceremoniales rituales en sociedades tempranas que aún se practican (Focacci y Pizarro 2000). Por tanto, con la migración y sus descendientes, las actividades de los sikuris se han transformado y ya no son estrictamente rituales ni se realizan de acuerdo con los mismos cánones de conducta y sistemas de significado. En Arequipa, estos grupos participan en pasacalles, marchas políticas, actividades académicas, lo que constituye un conjunto de funcionalidades distintas a las que se observan en las zonas de origen en el altiplano puneño. Al cambiar la funcionalidad, cambia el procedimiento y estas modificaciones se nutren a su vez de prácticas y visiones locales, lo que ha ofrecido la posibilidad a la mujer de aparecer como ejecutante -y no solo danzante- de grupos de sikuris, e incluso conformar grupos exclusivamente femeninos que basan su trabajo y su existencia en discursos y miradas completamente diferentes, e incluso divergentes, de los empleados en los centros de origen. Nuevas ideologías, nuevos imaginarios e incluso nuevas identidades están en permanente creación y transformación mientras se incorporan estos usos que, a su vez, se hallan bajo permanente revisión y crítica.

El presente artículo es parte de una investigación desarrollada por las autoras en torno al fenómeno de los grupos sikuris femeninos en la ciudad de Arequipa desde el 2018, con especial énfasis en lo referente a comportamientos sociales esperados por el sexo biológico. En concreto, este artículo se enfoca en el estudio de discursos, conductas y prácticas de la primera agrupación femenina, o 
agrupaciones mixtas con participantes femeninas en la ciudad.

\section{Metodología}

En Arequipa existen alrededor de quince agrupaciones de sikuris. Muchas de ellas funcionan como bases, es decir, como "sucursales" de agrupaciones originarias de la ciudad de Puno, las que se replican en ciudades no altiplánicas como Arequipa, Lima, Cusco e incluso en otros países. Se escogió como estudio de caso a la agrupación Awqa Sisa Warmy Sikuris por ser la primera agrupación exclusivamente femenina formada en la ciudad de Arequipa.

Para la recopilación de datos se realizaron entrevistas, observación participante y autoobservación. Juan Manuel Delgado y Juan Gutiérrez identifican "la autoobservación no solo por el hecho de que el observador pertenezca al grupo que investiga, sino porque se convierte en observador retrospectivo de una situación en que participó solo como actor y a la que se enfrentó con una actitud natural" (Delgado y Gutiérrez 1994:142). Así se deduce que los registros de información de los que el autoobservador se vale son no solo su propia memoria sino las notas, informes que produjo en su condición de actor y observante. Barbara Tedlock acerca de la observación participante indica que "La cuestión no es tanto la distancia, la objetividad y la neutralidad como la cercanía, la subjetividad y el compromiso" (Tedlok 1991:70), donde como investigadora se genera empatía con los sujetos de estudio, esto permite conversar con los sujetos, participar junto con ellos en diferentes prácticas y poder compartir y ser parte de la cotidianeidad del grupo.

Se realizaron veinte entrevistas etnográficas (estas entrevistas suelen ser abiertas y profundas, siendo este un lugar donde se construye conocimiento) aproximadamente, dentro de las que estaban las integrantes de Awqa Sisa, algunas mujeres sikuris que tocan en agrupaciones mixtas y hombres sikuris; fueron entrevistas profundas y reiterativas en su mayoría para conocer su percepción y discursos respecto de la mujer dentro de estos espacios musicales.

La sistematización de datos se realizó transcribiendo las entrevistas y diarios de campo en una base de datos codificando categorías y subcategorías según el orden siguiente:

a. percepción masculina de la incorporación de la mujer en los grupos de sikuris, b. las percepciones y discursos de las mujeres al integrar estas agrupaciones, así como sus motivaciones y aspiraciones al integrar un grupo de sikuris específicamente femenino.

Se registraron también diferentes contextos, ya que la participación de las mujeres en el ejercicio musical sikuri se da en actividades distintas a las de los grupos masculinos. Mientras estos suelen presentarse en ocasiones rituales y festivas, las mujeres lo hacen en otras de muy distinta naturaleza como son: presentaciones de libro, festivales de comida, marchas y actividades ligadas a la comunidad feminista y LGBTI de la ciudad.

La recolección de datos se realizó entre 2018 y 2019. Los datos e informaciones fueron recopilados en diferentes eventos sociales, religiosos y políticos. Los datos procesados se obtuvieron de entrevistas abiertas y estos datos se cruzaron en la consulta bibliográfica expuesta en la introducción.

\section{Resultados}

\section{Narración histórica del grupo}

A mediados del siglo XX el rol de la mujer dentro de los grupos de sikuris se limitaba a acompañar a los conjuntos realizando labores logísticas, encargándose de los instrumentos, la alimentación y la vestimenta de los ejecutantes. En cambio, en la segunda década del siglo XXI se observa a las mujeres en la sección de danza de los grupos de sikuris. Según lo explica Sánchez, el paso de acompañante a imilla ${ }^{1}$ fue un hecho fortuito relacionado con los concursos que se realizaban en la ciudad de Puno:

Cuando en 1982 se realizaría la presentación de la danza "soldado palla palla", la que se ejecuta con un solo bombo y luego pasaría a interpretar sikuris con varios bombos, para esta dinámica se usaron a mujeres que entraron a dejar los bombos y luego se presentaron como elenco de danza, esta novedad causó revuelo y la premiación del conjunto (Sánchez 2010:319). A finales del siglo XX se produce la transición de danzantes a intérpretes, este cambio se visualiza en mayor porcentaje en ciudades fuera de la zona andina, como Lima y Arequipa y unas pocas en la región de Puno. A decir de Turino, las ejecutantes manifestaban una continuidad cultural, esto quiere decir que estas mujeres habían tenido un vínculo cultural con la cultura de origen en este caso de procedencia puneña (Turino 1992). Esto 
sucede bien porque son hijas de migrantes puneños o bien porque sus familiares están involucrados con la música andina o porque han vivido algún tiempo en la zona altiplánica. Al existir esta relación cultural eligen continuar con estas manifestaciones, si bien se hallan en la necesidad de adaptarlas, transformarlas, revisarlas o recrearlas.

En la ciudad de Arequipa, entre 2015 y 2016, cuatro mujeres ingresaron a tocar en agrupaciones masculinas cuyas experiencias sirven de base para comprender mejor el proceso de incursión femenina en este campo musical en la ciudad de Arequipa. Su ingreso en los grupos musicales fue por invitaciones de amigos y conocidos y no tuvieron problemas de recepción (discriminación o machismo).

Una de ellas llegó a ser parte de la junta directiva e incluso presidenta de la agrupación AJP Sikuris 27 de Junio. Su ingreso al grupo no tuvo contratiempos, y hubo un trabajo personal intenso para tocar el bombo en su agrupación, labor que era exclusivamente masculina. Manifiesta que como mujer solo le pasaban el siku malta ${ }^{2}$ para tocar, pero no las zanjas ni tollos que son para sikuris más experimentados y mucho menos el bombo. Debido a estas restricciones decidió iniciar una práctica junto con otra compañera para estimular su propio avance. Estas restricciones provocaron una notoria diferencia entre su progreso y el de los integrantes masculinos, cuyo progreso es más ordenado, sistemático y seriado desde la condición de malta hasta la condición de bombero ${ }^{3}$ y ocasionan que las mujeres busquen y generen sus propias experiencias de aprendizaje, a diferencia de los varones cuyos procesos didácticos dentro del grupo son más colectivos y organizados.

Otra de las dificultades que tienen estas mujeres dentro de las agrupaciones masculinas es que existe una creencia colectiva que los sikuris están relacionados al consumo abusivo de bebidas alcohólicas y una vida bohemia intensa, actividades que, por estereotipo de género, se relacionan solo a los hombres mas no a mujeres. Blanca Gonzales define los estereotipos como "creencias consensuadas sobre las diferentes características de los hombres y mujeres en nuestra sociedad". Este conjunto de creencias que atañen a las categorías de hombre y mujer tiene una gran influencia en el individuo, en su percepción del mundo y de sí mismo y en su conducta (González 1999:84). Es así como estas creencias dificultan la integración de las mujeres en los grupos de sikuris de hombres, ya que, según los estereotipos de género, ellas no deben estar a la par en cuanto a beber licor con hombres ni mucho menos asistir a actividades a altas horas de la noche fuera de sus hogares. Varias entrevistadas manifiestan que cuando asistían a eventos y festividades nocturnas con sus pares masculinos tenían que tomar precauciones para evitar acciones impropias de sus compañeros provocadas por la ingesta de bebidas alcohólicas.

La mayoría de los hombres entrevistados están de acuerdo con la participación de mujeres en sus agrupaciones. Ven que esta integración aporta "frescura y novedad al grupo", sin embargo, aclaran que no van a tener la fuerza (ya sea física o psicológica) ni continuidad musical de los hombres, ellos han observado que varias mujeres integraron por un tiempo sus agrupaciones, pero se retiraron porque no pueden sacrificar su responsabilidad al momento de formar una familia como los hombres sikuri sí pueden hacerlo.

Awqa Sisa Warmy Sikuris es la primera agrupación de sikuris conformada por mujeres fundada en la ciudad de Arequipa y que no tiene correspondencia con grupos de otras ciudades, como suelen conformarse los grupos masculinos. Se formó a principios del 2017 con la iniciativa de dos de aquel cuarteto femenino que ya tenían experiencia en sikuris masculinos, quienes publicaron convocatorias en las redes sociales y entre sus amistades y lograron reunir entre siete a doce mujeres. Sus integrantes vienen de distintas ciudades sur peruanas (Puno, Arequipa, Cusco) y algunas de ellas pertenecen a la comunidad LGBTI. Las dos fundadoras, de las que una dejó el grupo en 2019, son las únicas que iniciaron su trayectoria musical en agrupaciones de hombres, las demás integrantes tuvieron su primer contacto musical sikuri con las mujeres de Awqa Sisa.

Las integrantes de este grupo, a diferencia de sus pares masculinos, han participado como invitadas en diversos eventos culturales, presentaciones de libros, conversatorios y otras actividades donde no suele ser común la presencia de grupos sikuris. Gracias a la atención captada en estas actividades, a principios del 2019 se observó una mayor afluencia de integrantes. Tuvo especial éxito su participación en la marcha del 8 de marzo por el día de la mujer y lograron convocar más de una docena de mujeres instrumentistas. En dicha ocasión, un grupo de imillas las acompañaron bailando e incluso invitaron a integrantes masculinos de otras agrupaciones de 
sikuris hasta llegar a reunir seis participantes varones. Una fecha de tal trascendencia llevó a que el material audiovisual producto de esta intervención se propague por las redes sociales (Facebook y youtube, principalmente). La difusión masiva de estos materiales sirvió para que más mujeres interesadas participaran en los ensayos y presentaciones estimulando el crecimiento del grupo.

\section{Discursos y comportamientos de las mujeres en los grupos sikuris}

El comportamiento y desenvolvimiento de este grupo sikuri encuentra correspondencia con lo descrito por Judith López Uruchi, Antropóloga y directora del grupo de mujeres Sikuri Inalmama, cuando explica que para ella es una forma de resistencia cultural, las mujeres difunden la música según sus necesidades y el contexto en el que se encuentran, por esta razón es que se ha visto en los últimos años grupos de mujeres sikuris feministas en las zonas urbanas.

Las prácticas colectivas de estas mujeres es contenerse emocionalmente cuando se reúnen en círculos. No nos vamos a medir con los varones porque ellos tienen una manera de expresar muy diferente a la nuestra. Ellos desde su masculinidad, desde su fuerza física. Nosotras nos expresamos de distinta manera la forma de hacer música a través de nuestro sentir, nuestro canto, a través de nuestros soplidos (López 2019).

Asimismo, como exponía Turino, la música es frecuentemente escogida como signo semiótico central por las unidades de identidad en sus estrategias sociales. Cuando es usada en esta tarea, la música misma puede llegar a ser una fuerza de cambios sociales (Turino 1998). El papel que juega la música en la creación de identidades es una cuestión central para las investigaciones respecto de las llamadas músicas populares urbanas, y más aún cuando se incorpora a ellas la perspectiva de género. Para Karen Avenburg las expresiones musicales son fenómenos culturales que proporcionan elementos que se pueden utilizar en la construcción de su identidad colectiva (Avenburg 2012). Con base en esto, se puede entender por qué las integrantes de Awqa Sisa manifiestan que no solo quieren despertar sentimientos en el público demostrando ser la única agrupación femenina en Arequipa y ocupando espacios músico-culturales, sino que también usan la música para realizar reivindicaciones expresivas, laborales y de interrelación artística con sus pares masculinos, empoderándose mediante ideales feministas y teniendo lazos con varios colectivos en la ciudad, por lo que, a diferencia de las agrupaciones masculinas, ellas se dan a conocer en marchas, conversatorios, presentaciones de libros y demás actividades relacionadas con expresiones entendidas como feministas.

Carrasco y Gavilán han observado que para identificar e interpretar los discursos feministas de las mujeres es necesario ver el contexto donde se desarrollan. Este discurso como práctica social incluye ideas y sentidos que tienen para los actores sus comportamientos; es decir, las interpretaciones que hacen de sí mismos y de su entorno (Carrasco y Gavilan 2017). No todas las integrantes se identifican con la orientación feminista de los discursos del grupo. En 2019, dos integrantes optaron por retirarse de la agrupación al no compartir la orientación ideológica del mismo. En el caso de una de ellas, que fue invitada a participar por sus amigas, existe continuidad cultural en su práctica debido a que uno de sus abuelos y otros familiares incluyendo su actual pareja, son músicos y forman parte del movimiento sikuri. Su motivación para el ingreso al grupo fue por aprender a tocar y manifiesta que: "El sikuri no es ideología, sino música y pasión". Este interés en la práctica, mas no en la ideología, fue lo que la llevó a dejar la agrupación e integrar una de varones. A diferencia de otras integrantes, su formación musical empezó con mujeres sikuris, lo que hace que la integración con su actual agrupación masculina sea más complicada debido a la disparidad de metodologías empleadas en ambos tipos de grupos, que se explican más adelante. Lo primero que se le dificulta es el aprendizaje, ya que se acostumbró al método dinámico de ensayo practicado por las mujeres, mientras que los hombres tienen un método menos intensivo y un repertorio más amplio.

Lorena Cabnal ha señalado que "La construcción de epistemología feminista comunitaria, afirma que existe patriarcado originario ancestral, que es un sistema milenario estructural de opresión contra las mujeres originarias o indígenas" (Cabnal 2010:121). Así, se reinterpretan las realidades de la vida histórica y cotidiana de las mujeres indígenas, dentro del mundo indígena y fuera de el en espacios 
urbanos por medio de algunas performances de las mujeres sikuris. Además, los estudios de Silvia Rivera Cusicanqui revelan que la idea que cuando se dice que lo occidental es universal es una percepción dominante, que llega a confinar hábitos, cosmovisiones, tradiciones a un ámbito específico local y folclórico, destinado a no reproducirse en las próximas generaciones (Rivera 2003), menos aún reproducirse en lo urbano y quien lo haga está transgrediendo ciertas verdades que deben mantenerse en un ámbito específico. Es por ello que Silvia Vega asevera que "las organizaciones feministas tradicionales han asumido la interpelación a la construcción intercultural del feminismo en el nuevo contexto y a la ampliación de sus agendas históricas, incorporando temas que provienen de las otras dominaciones sociales además de la de género" (Vega 2014:88). Estas discursividades feministas comunitarias se contraponen al discurso occidental, razón por que se hace un intento por reconocer la historia de América Latina para construir un pensamiento feminista de acuerdo con sus contextos, demandas y urgencias.

El nombre de la agrupación Awqa Sisa Warmy Sikuri de por sí ya excluye la integración de hombres y esta es la queja que tienen varios varones sikuris que manifiestan que en los grupos tradicionales pueden ingresar mujeres, pero en estos nuevos grupos ellos se sienten excluidos. Uno de los entrevistados comenta estar de acuerdo con que las mujeres toquen pero que si quieren revalorar a la mujer andina deberían vestirse como tal, "los hombres revaloramos la vestimenta del hombre con pantalón de bayeta, la camisa, etc. Pero las mujeres tienen una contradicción al vestirse con pantalones y polleras encima, en algunas presentaciones". Awqa Sisa para él "es un buen proyecto sobre igualdad de género y en el aspecto social está bien, pero en lo cultural no está de acuerdo porque se tergiversa el sentido sikuri y no respetan las tradiciones".

Para algunos hombres el empoderamiento de las mujeres se basa en excluir a los hombres del espacio y acción musical, lo que es un concepto erróneo. Rita Segato, en una entrevista, manifestó que "empoderarse es otra palabra que no utilizo, porque cuando uno habla de poder implica despoder y a mí me interesa aspirar a un mundo horizontal". (Segato 2019). Los hombres no le temen a esa horizontabilidad del poder porque confían en mantenerlo mientras que la mujer está acostumbrada a ser desplazada.

\section{Procedimientos de enseñanza-aprendizaje}

El proceso de enseñanza-aprendizaje de las mujeres respecto de los hombres es distinto y como ya se ha explicado, ellas forman un círculo de confianza y apoyo grupal, pero su ejercitamiento es más individual que el realizado en los grupos masculinos debido, dicen, a la presión existente de transgredir una ley no escrita de que la mujer no debe tocar. Esto hace que sean más detallistas y perfeccionistas en el estudio ante el temor de la recepción de su trabajo por sus pares masculinos, lo que lleva a que refuercen más sus ensayos en solitario y en sus propios espacios. No priorizan los aprendizajes colectivos o en pareja que es muy importante al momento de tocar sikuri en los grupos masculinos.

Acerca de la forma de tocar colectiva, el etnomusicólogo Américo Valencia manifiesta que tocar en grupo es "confeccionar frases musicales que componen una melodía nota a nota". Para lograr esto se necesita un diálogo íntimo tanto grupal como en pareja y lograr una alternancia de sonidos hechos por la pareja de sikus que van a formar una sola unidad (Valencia 1989: 61). Podhajcer indica que la ronda, el diálogo y el "tocar juntos" constituyen el carácter icónico-indexical como ícono de lo comunitario, que se va "cargando" de emociones en cada cita, en cada performance. Esta unidad social es asimismo una fuerza musical que la audiencia percibe (Podhajcer 2015:51). Este trabajo de la pareja musical está ausente en los ensayos de Awqa Sisa, ya que priorizan los aprendizajes individuales.

Para los entrevistados de ambos sexos, los hombres fueron los primeros en tocar sikuri, por lo que las mujeres siempre van a depender de ellos para aprender o formar una agrupación. Ellos han percibido que esto sucede con la agrupación Awqa Sisa en la que hay un hombre que las acompaña en los ensayos, él se siente orgulloso de la agrupación por la pasión y empeño de las mujeres en aprender, pero no se siente parte del grupo, "jamás seré una Warmy (mujer)" dice.

Otra estrategia es la que emplean en el aprendizaje del siku: en la mayoría de grupos masculinos aprenden a tocar por imitación sin el uso de sistemas gráficos ni simbólicos escritos. Entre estos sistemas se encuentra la numerofonía cuyo uso es minimizado por los grupos masculinos que prefieren darles énfasis a los aprendizajes colectivos. Las mujeres 
emplean activamente el sistema numerofónico para el aprendizaje musical.

\section{Discusión}

Por el trabajo de campo realizado, las observaciones y entrevistas llevadas a cabo con los grupos elegidos se pueden concluir varias ideas:

Se ha encontrado que las mujeres identifican algún tipo de conflicto cuando participan en agrupaciones masculinas. Estas dificultades se dan en el aspecto del aprendizaje (los niveles de dificultad cuando aprenden, las metodologías de enseñanza, la difusión de los contenidos), así como la imposibilidad de participar en todas las actividades de las agrupaciones en igualdad de condiciones que los varones. Esto se debe, sobre todo, a la presencia de estereotipos de los roles de género que, muy a menudo, impiden la permanencia a largo plazo de los elementos femeninos.

El contraer matrimonio significa una restricción severa para sus actividades a futuro, por tanto las mujeres no siempre resultan confiables y elegibles en los grupos tradicionales porque a la larga tienden a dejar el grupo, esta imposibilidad de un proyecto a largo plazo en agrupaciones masculinas y los problemas generados por el proceso de enseñanza, o asimilación de las técnicas del sikuri, ha sido motivo de que se formen agrupaciones femeninas con sus propios mecanismos de selección, enseñanza, ensayo, transmisión de los contenidos y actuaciones. En el caso de la agrupación femenina Awqa Sisa Warmy Sikuris la transmisión de conocimientos se realiza de mujer a mujer con el uso del método de numerofonías. Los ensayos tienden a ser repetitivos y constantes a lo largo de cada mes, lo que reduce el tamaño del repertorio que practican en comparación con el de los grupos masculinos que es más extenso. Por contraparte, sus presentaciones abarcan contextos culturales distintos a los de sus pares masculinos como marchas, presentaciones de libros y conferencias.

Este grupo tiene la particularidad de priorizar instancias de ensayo que muchas veces no tienen como objetivo la exhibición o actuación en un escenario, la grabación de un disco o la militancia política. A diferencia de los grupos de sikuris conformados por hombres, que participan en contextos más tradicionales de los sikuris, Warmi Sikuris construye relaciones contextuales nuevas y distintas a aquellas que suelen desarrollar los grupos de sikuris de varones. Existen casos en los que mujeres que conformaron grupos de mujeres sikuris regresaron a sus grupos mixtos de origen porque expresaron comodidad y son bien recibidas estableciendo una relación interesante con el grupo original y los nuevos grupos formados.

La continuidad cultural es un factor importante que influye en el discurso de las mujeres al momento de escoger el grupo en el que van a tocar. Aquellas que no tienen esta experiencia previa se han iniciado tocando con mujeres, mientras que las que la poseen se sienten más cómodas tocando con hombres. Esto puede indicar que la incorporación de intérpretes que no tienen experiencia puede ser por medio de mujeres y no necesariamente de hombres, lo que supone una apertura en los roles de género para la transmisión de conocimientos y adecuación de usos y comportamientos musicales. Awqa Sisa Warmy Sikuri ha abierto una nueva frontera en el aspecto de aprendizaje, los hombres ya no monopolizan la enseñanza y transmisión de la tradición sikuri.

Los hombres usan estereotipos de género en sus discursos para argumentar que la mujer no posee continuidad musical en el grupo debido a sus labores dentro de la familia, mientras que ellos no tienen (el rol masculino no los obliga a ello) necesidad de alejarse por el cuidar de la familia. Los roles masculino y femenino, de lo que se puede y no se puede hacer con relación con el estado civil siguen siendo bastante diferenciados para hombres y para mujeres entre los cultores de sikuris y es un factor que determina la permanencia o no del músico en la agrupación. En la mayoría de agrupaciones, la actividad no supone un beneficio económico. En la eventualidad de que se produzcan algunos contratos, los beneficios se destinan a comprar instrumental nuevo, reparar el existente o adquirir nueva indumentaria. No es posible para un integrante depender económicamente de su actuación en el conjunto.

La vestimenta durante las presentaciones es el escenario metafórico de conflicto soterrado. Las mujeres de Awqa Sisa usan polleras y, al hacerlo, manifiestan conscientemente una reivindicación de la mujer andina de las comunidades originarias, identificándose con estas causas, aunque esté descontextualizada. La práctica sikuri andina en ciertas comunidades tradicionales se mantiene lejos de su centro de origen y el emplear códigos de vestimenta considerados propios de comunidades 
que dieron origen a la práctica sikuri, supone una paradoja relevante, ya que en estas comunidades la participación de las mujeres en las tropas sikuris está restringida. De esta forma, se intenta reivindicar a la mujer andina que no puede participar en otro entorno, donde incluso se recurre al discurso feminista para completar esta reivindicación.

Respecto del uso de este término, algunos conjuntos lo rechazan, como lo ha manifestado Judith López directora del grupo de mujeres Sikuri Inalmama. En el marco del Tercer Congreso Internacional de Sikuris en Buenos Aires 2019, López recalcaba que su agrupación no es feminista, "ya que esas prácticas feministas vienen de Occidente", por tanto, en su agrupación como expresión de resistencia cultural las mujeres andinas visten de acuerdo con sus trajes identitarios. Si la resistencia cultural que las induce a utilizar los trajes identitarios fuera tan consistente, no habría mujeres actuando en tropas sikuris en contextos nuevos. De esto se deduce que las mujeres seleccionan, conscientemente, los discursos y los elementos que les resultan más útiles y necesarios para sus desempeños en los conjuntos formados bajo circunstancias diferentes, donde aprovechan espacios y oportunidades nuevas.

$\mathrm{Al}$ apelar a la "originalidad" de la tradición, en el caso Awqa Sisa, manifestaron que no intentan recrearla, sino que desean ganar legitimidad vinculándose con ella. Desean que se les reconozca por ser tradicionales, no porque quieran serlo. La legitimación de sus prácticas proviene de su público, sus pares masculinos y de otros grupos receptores, pero resulta paradójico y contradictorio con su discurso feminista. La expresión de este discurso se realiza, en cierto modo, al no legitimar lo que se hace apegándose a la tradición sino lograr reconocimiento precisamente por renunciar a ella.

\section{Agradecimientos}

A las agrupaciones de sikuris de Arequipa en especial Awqa Sisa Warmy con quienes se realizó el trabajo de campo, así como también a la red de sikuris investigadores por su apoyo durante la investigación. A los evaluadores anónimos de este artículo por sus comentarios y sugerencias que ayudaron a enriquecer el artículo.

\section{Referencias Citadas}

Avenburg, K

2012 "Interpellation and Performance. The Construction of Identities through Musical Experience in the Virgen del Rosario Fiesta in Iruya, Argentina". Latin American Perspectives 39 (2): 134-149.

Delgado, J y Gutiérrez

1995 Métodos y Técnicas Cualitativas de Investigación en Ciencias Sociales. Madrid.

Del Pino, P.

2017 En Nombre del Gobierno El Perú y Uchuraccay, Un siglo de Política Campesina. La siniestra ensayos, Juliaca.

Cabnal, L.

2010 "Acercamiento a la construcción de la propuesta de pensamiento epistémico de las mujeres indígenas feministas comunitarias de Abya Yala". Momento de paro Tiempo de rebelión, 116-134.

Carrasco, A. y Gavilán, V.

2014 "Género y etnicidad: Ser hombre y ser mujer entre los aymara del altiplano chileno". Diálogo Andino 45:169-180.

Chacama J y A, Díaz

2011 "Cañutos y soplidos tiempo y cultura en Las Zampoñas de las sociedades precolombinas de Arica". Revista Musical Chilena, 216:34-57.

Echenique, A.

1997 "Género y estructuras de poder en la cultura andina". Estudios: Centro de estudios avanzados 7:159-172.

Focacci, G. Pizarro, E.

2000 "Relatos de cronistas sobre instrumentos musicales andinos. Presencia de ellos en la arqueología de Arica". Revista Diálogo Andino, 19:33-50.
Gargallo, F.

2015 Feminismos desde Abya Yala: ideas y proposiciones de las mujeres de 607 pueblos en nuestra América. Universidad Autónoma de la Ciudad de México.

Gavilán, V y Carrasco, A.

2018 "Prácticas discursivas e identidades de género de las mujeres aymaras del norte chileno (1980-2015)". Diálogo Andino 55:111-120.

González, Blanca

1999 "Los estereotipos como factor de socialización en el género". Revista Comunicar 12: 79-88.

López, J.

2019 Mujeres Sikuris y Prácticas Emancipatorias: deconstruyendo estereotipos. Ponencia presentada en $3^{\circ}$ Congreso Internacional de Sikuris, Buenos Aires, Argentina.

Mamani, M.

2010 "Kirkir Warmi: identidad y rol de la mujer aymara en el desarrollo musical del norte". Revista Musical Chilena 64:90-102.

Mardones, P y Moñino, I.

2020 "Música, fronteras y etnogénesis sikuri. El ascenso musical al Abra de Punta Corral, Tilcara (Jujuy, Argentina)". Resonancias 24:99-131.

Mendívil, J.

2016 En Contra de la Música. Gourmet Musical, Buenos Aires.

Podhajcer, A.

2015 "Sembrando un cuerpo nuevo: Performance e interconexión en prácticas musicales 'andinas' de Buenos Aires". Revista Musical Chilena 69: 47-65. 
Podhajcer, A.

2008 Jjaktasiña irampi arcampi: emoción y creencias en la creatividad de conjuntos de "música andina" de Buenos Aires y Puno (Perú). IX Congreso Argentino de Antropología Social. Facultad de Humanidades y Ciencias Sociales Universidad Nacional de Misiones, Posadas.

Rivera S.

2003 Las fronteras de la coca: epistemologías coloniales y circuitos alternativos de la hoja de coca: el caso de la frontera boliviano-argentina. La Paz, Bolivia: IDIS, UMSA, Ediciones Aruwiyiri.

Rodríguez, M.

2017 "Mujeres indígenas y sistema de cargos en el siglo XXI. México". Diálogo Andino-Revista de Historia, Geografía y Cultura Andina 52: 45-55.

Sánchez, C.

2014 Organización, arte, identidad e ideología en los grupos de sikuris metropolitanos: Procesos sociales y de cultura juvenil en lima (1980-2000). Tesis para optar el grado académico de magíster en antropología, Universidad Nacional de San Marcos, Lima, Perú.

Segato, R.

2007 La nación y sus otros: raza, etnicidad y diversidad religiosa en tiempos de políticas de la identidad. Prometeo Libros Editorial. Un acercamiento desde la Sierra de Zongolica, Veracruz,

Segato, R.

2019 La antropóloga que incomoda. (18 setiembre). http:// revistaanfibia.com/cronica/la-antropologa-que-incomoda (15 noviembre 2018).

Suárez, L.

2003 "La construcción de las identidades de género en la música popular". Dossiers feministes 11-31.

Tedlock, B.

1991 "De la observación participante a la observación de la participación: el surgimiento de la etnografía narrativa", Journal of Anthropological Research 47: 69-94.

Turino, T.

1992 "Del esencialismo a lo esencial: Pragmática y significado de la interpretación de los sikuris puneños en Lima". Revista Andina 2: 441-456.

Turino, T.

1998 "La coherencia del estilo social y de la creación musical entre los Aymara del sur del Perú". Música, Danzas y Máscaras en los Andes Editado por R. Romero pp. 61-95. PUCP, \& Instituto Riva-Agüero, Lima, Perú.

Valencia, A.

1989 El Siku Altiplánico. Estudio de los Conjuntos Orquestales de Sikus Bipolares Del Altiplano Peruano. Casa de las Américas, Habana.

\section{Vega, S.}

2014 "El orden de género en el sumak kawsay y el suma qamaña: un vistazo a los debates actuales en Bolivia y Ecuador". Íconos. Revista de Ciencias Sociales 48:73-91.

\section{Notas}

1 El término imilla se refiere a la joven andina, pero en los grupos de sikuris es tomado como las bailarinas que acompañan los grupos de sikuri.

2 Por maltas se entiende a los sikus de tamaño y sonido medio, son la base sonora de la melodía y los ejecutantes se inician tocando malta mientras que la zanja es de mayor tamaño y con el sonido fuerte dentro de la tropa de instrumentos.

3 Bombero es el sikuri que toca el bombo en la agrupación. 